\title{
OPEN Subclinical gait disturbance and postoperative gait improvement in patients with degenerative cervical myelopathy
}

\author{
Dong-Ho Lee ${ }^{1}$, Jong Yoon $\mathrm{Yoo}^{2}$, Jae Hwan Cho ${ }^{1}$, Chang Ju Hwang ${ }^{1}$, Choon Sung Lee ${ }^{1}$, \\ Chunghwan Kim ${ }^{3}$, Jung-Ki Ha ${ }^{3}$ \& Kun-Bo Park ${ }^{4}$
}

This study aimed to evaluate the subclinical gait abnormalities and the postoperative gait improvements in patients with degenerative cervical myelopathy using three-dimensional gait analysis. We reviewed the gait analysis of 62 patients who underwent surgical treatment for degenerative cervical myelopathy. The asymptomatic gait group included 30 patients and the gait disturbance group included 32 patients who can walk on their own slowly or need assistive device on stairs. The step width $(17.2 \mathrm{~cm}$ vs. $15.9 \mathrm{~cm}, P=0.003)$, stride length $(105.2 \mathrm{~cm}$ vs. $109.1 \mathrm{~cm}, P=0.015)$, and double-limb support duration $(13.4 \%$ vs. $11.7 \%, P=0.027)$ improved only in the asymptomatic gait group. Preoperatively, the asymptomatic gait group exhibited better maximum knee flexion angle $\left(60.5^{\circ}\right.$ vs. $\left.54.8^{\circ}, P=0.001\right)$ and ankle plantarflexion angle at push-off $\left(-12.2^{\circ} \mathrm{vs} .-6.5^{\circ}\right.$, $P=0.001$ ) compared to the gait disturbance group. Postoperatively, maximum knee flexion angle $\left(62.3^{\circ}\right.$ vs. $\left.58.2^{\circ}, P=0.004\right)$ and ankle plantarflexion angle at push-off $\left(-12.8^{\circ} \mathrm{vs} .-8.3^{\circ}, P=0.002\right)$ were still better in the asymptomatic gait group, although both parameters improved in the gait disturbance group ( $P=0.005,0.039$, respectively). Kinematic parameters could improve in patients with gait disturbance. However, temporospatial parameters improvement may be expected when the operative treatment is performed before apparent gait disturbance.

Various degenerative conditions of the cervical spine, including cervical spondylotic myelopathy, degenerative disc disease, and ossification of the posterior longitudinal ligament and ligamentum flavum can result in degenerative cervical myelopathy $(\mathrm{DCM})^{1}$. There are several manifestations of DCM, including clumsy hands, paresthesia, neck pain, and gait disturbances ${ }^{2}$. Gait disturbances in DCM are caused by a spinal cord impairment lesion; the severity of gait disturbance is diverse and should be systematically examined, as they could be misunderstood as a symptom of lumbar spinal disease ${ }^{3-5}$. However, functional scales (GRASSP-M, Nurick, and JOA) designed to evaluate disability in patients with DCM provide only qualitative information of gait disability, rather than quantitative assessment, and may not be sensitive to less pronounced gait changes since a single category often encompasses a wide range of severities or do not include gait abnormality ${ }^{6-8}$.

Three-dimensional gait analysis can provide detailed and quantifiable information about gait parameters. Previous studies suggested slow walking speed or reduced joint range of motion (ROM) as pathologic gait parameters in DCM, and reported an improvement of gait parameters after operation ${ }^{9-11}$. However, the decrease in gait parameters was diverse, and Malone et al. found that temporospatial or other kinematic parameters showed no improvement after operation ${ }^{9,12}$. This inconsistent result may be related to the different severity of DCM in terms of gait disturbance. Patients may have only upper extremity symptoms and may not be aware of their gait disability, or the severely affected gait disturbance may not improve after operation ${ }^{2,13,14}$. Identifying quantitative

\footnotetext{
${ }^{1}$ Department of Orthopedic Surgery, Asan Medical Center, University of Ulsan College of Medicine, Seoul, Korea. ${ }^{2}$ Department of Rehabilitation Medicine, Asan Medical Center, University of Ulsan College of Medicine, Seoul, Korea. ${ }^{3}$ Department of Orthopedic Surgery, Gangneung Asan Hospital, University of Ulsan College of Medicine, Gangneung, Korea. 'Division of Pediatric Orthopedic Surgery, Severance Children's Hospital, Yonsei University College of Medicine, 50-1 Yonsei-ro, Seodaemun-gu, Seoul 03722, Korea. ${ }^{\varpi}$ email: pedoskbp@yuhs.ac
} 


\begin{tabular}{|l|l|l|l|}
\hline Characteristics & Asymptomatic gait & Gait disturbance & $P$ value \\
\hline Age (years) & $61.4 \pm 10.8$ & $55.1 \pm 9.0$ & 0.437 \\
\hline Duration of symptoms before surgery (months) & $11.5 \pm 4.3$ & $12.3 \pm 5.1$ & 0.553 \\
\hline Sex (Male:Female) & $22: 8$ & $24: 8$ & 0.162 \\
\hline Body mass index $\left(\mathrm{kg} / \mathrm{m}^{2}\right)$ & $27.4 \pm 6.5$ & $27.8 \pm 10.8$ & 0.860 \\
\hline JOA scores & $14.6 \pm 1.9$ & $11.6 \pm 2.3$ & $0.001^{*}$ \\
\hline Preoperative & $15.1 \pm 1.7$ & $12.4 \pm 3.0$ & $0.001^{*}$ \\
\hline Postoperative & \multicolumn{5}{|l}{} \\
\hline Operation & 19 & 21 & \multirow{2}{|}{0.530} \\
\hline Anterior & 11 & 11 & \\
\hline Posterior & \multicolumn{2}{|l}{}
\end{tabular}

Table 1. Patient characteristics of asymptomatic gait and gait disturbance groups. JOA Japanese Orthopedic Association. ${ }^{\star} P<0.05$.

\begin{tabular}{|l|l|l|l|}
\hline Parameters & Asymptomatic gait & Gait disturbance & P value \\
\hline Preoperative & $17.2 \pm 3.4$ & $17.4 \pm 2.4$ & 0.553 \\
\hline Step width (cm) & $105.2 \pm 11.5$ & $94.2 \pm 23.4$ & $0.001^{*}$ \\
\hline Stride length (cm) & $93.9 \pm 13.6$ & $81.5 \pm 24.9$ & $0.001^{*}$ \\
\hline Velocity (cm/s) & $61.8 \pm 4.6$ & $64.2 \pm 5.7$ & $0.001^{*}$ \\
\hline Duration of standing phase (\% of gait cycle) & $13.4 \pm 3.7$ & $16.1 \pm 7.3$ & $0.011^{*}$ \\
\hline Duration of double-limb support (\% of gait cycle) & & $18.7 \pm 8.8$ & $0.021^{*}$ \\
\hline Postoperative & $15.9 \pm 3.4$ & $97.2 \pm 23.7$ & $0.001^{*}$ \\
\hline Step width (cm) & $109.1 \pm 15.2$ & $85.3 \pm 24.9$ & $0.008^{*}$ \\
\hline Stride length (cm) & $96.7 \pm 22.1$ & $63.3 \pm 3.9$ & $0.003^{*}$ \\
\hline Velocity (cm/s) & $61.3 \pm 3.2$ & $14.8 \pm 9.2$ & $0.016^{*}$ \\
\hline Duration of standing phase (\% of gait cycle) & $11.7 \pm 4.3$ & & \\
\hline Duration of double-limb support (\% of gait cycle) & &
\end{tabular}

Table 2. Comparison of temporospatial parameters between asymptomatic gait and gait disturbance groups. ${ }^{\star} P<0.05$.

gait differences in patients with DCM would contribute to the detection of subtle preoperative impairments and help predict postoperative improvements.

In this study, we reviewed pre- and postoperative gait analysis of DCM patients who had gait disturbance and those without gait symptoms. The current study aimed to evaluate (1) preoperative subclinical gait abnormalities in patients with DCM and (2) the improvements in gait parameters after surgery using three-dimensional gait analysis in patients with and without apparent gait abnormality.

\section{Results}

Participants. Between asymptomatic gait and gait disturbance groups, there was no difference in the age at surgery $(61.4 \pm 10.8$ years vs. $55.1 \pm 9.0$ years, $P=0.437)$, duration of preoperative symptoms $(11.5 \pm 4.3$ months vs. $12.3 \pm 5.1$ months, $P=0.553)$, and anterior/posterior operation $(19 / 11 \mathrm{vs.} 21 / 11, P=0.53)$. Both pre- $(14.6 \pm 1.9$ vs. 11.6 \pm 2.3$)$ and postoperative $(15.1 \pm 1.7$ vs. $12.4 \pm 3.0)$ JOA scores were higher in the asymptomatic gait group (Table 1). JOA scores improved significantly after surgery in both groups $(P=0.013$ for asymptomatic gait and $P=0.016$ for gait disturbance group). However, there was no significant change in lower limb motor function grade according to JOA ( $P=0.083$ for asymptomatic gait and $P=0.231$ for gait disturbance group).

Comparison of temporospatial parameters between groups. Preoperatively, patients in the gait disturbance group exhibited a shorter stride length $(105.2 \pm 11.5 \mathrm{~cm}$ vs. $94.2 \pm 23.4 \mathrm{~cm}, P=0.001)$ and a slower walking speed $(93.9 \pm 13.6 \mathrm{~cm} / \mathrm{s}$ vs. $81.5 \pm 24.9 \mathrm{~cm} / \mathrm{s}, P=0.001)$, in addition to their longer standing phase duration $(61.8 \pm 4.6 \%$ vs. $64.2 \pm 5.7 \%, P=0.001)$ and double-limb support duration $(13.4 \pm 3.7 \%$ vs. $16.1 \pm 7.3 \%, P=0.011)$, compared to the asymptomatic gait group. However, there was no difference in the step width $(17.2 \pm 3.4 \mathrm{~cm}$ vs. $17.4 \pm 2.4 \mathrm{~cm}, P=0.553)$.

Postoperatively, patients in the gait disturbance group continued to have a shorter stride length $(109.1 \pm 15.2 \mathrm{~cm}$ vs. $97.2 \pm 23.7 \mathrm{~cm}, P=0.001)$ and slower walking speed $(96.7 \pm 22.1 \mathrm{~cm} / \mathrm{s}$ vs. $85.3 \pm 24.9 \mathrm{~cm} / \mathrm{s}$, $P=0.008)$ with longer standing phase duration $(61.3 \pm 3.2 \%$ vs. $63.3 \pm 3.9 \%, P=0.003)$ and double-limb support duration $(11.7 \pm 4.3 \%$ vs. $14.8 \pm 9.2 \%, P=0.016)$. Furthermore, postoperative step width became longer compared to the asymptomatic gait group $(15.9 \pm 3.4 \mathrm{~cm}$ vs. $18.7 \pm 8.8 \mathrm{~cm}, P=0.021)$ (Table 2). 


\begin{tabular}{|l|c|c|l|}
\hline Parameters & Asymptomatic gait & Gait disturbance & $P$ value \\
\hline Kinematics $\left(^{\circ}\right)$ & $9.0 \pm 6.0$ & $9.9 \pm 8.2$ & 0.493 \\
\hline Mean pelvic anterior tilt angle & $-4.1 \pm 14.5$ & $-2.9 \pm 13.9$ & 0.660 \\
\hline Maximum hip extension angle & $60.5 \pm 6.4$ & $54.8 \pm 9.5$ & $0.001^{*}$ \\
\hline Maximum knee flexion angle & $-12.2 \pm 7.4$ & $-6.5 \pm 6.5$ & $0.001^{*}$ \\
\hline Ankle plantarflexion angle at push-off & $-3.0 \pm 4.3$ & $-2.6 \pm 3.7$ & 0.502 \\
\hline Ankle plantarflexion angle at initial contact & & \\
\hline Kinetics & $0.9 \pm 0.4$ & $0.9 \pm 0.4$ & 0.398 \\
\hline Maximum ankle moment (Nm/kg) & $1.1 \pm 0.7$ & $1.3 \pm 3.1$ & 0.637 \\
\hline Maximum ankle power generation $(\mathrm{W} / \mathrm{kg})$ &
\end{tabular}

Table 3. Comparison of preoperative kinetic and kinematic parameters between groups. Negative values indicate hip extension and ankle plantarflexion. ${ }^{\star} P<0.05$.

\begin{tabular}{|l|c|c|l|}
\hline Parameters & Asymptomatic gait & Gait disturbance & $P$ value \\
\hline Kinematics $\left(^{\circ}\right)$ & $10.6 \pm 6.1$ & $11.6 \pm 7.7$ & 0.455 \\
\hline Mean pelvic anterior tilt angle & $-3.8 \pm 8.9$ & $0.7 \pm 16.2$ & 0.057 \\
\hline Maximum hip extension angle & $62.3 \pm 5.4$ & $58.2 \pm 9.7$ & $0.004^{*}$ \\
\hline Maximum knee flexion angle & $-12.8 \pm 8.2$ & $-8.3 \pm 8.1$ & $0.002^{*}$ \\
\hline Ankle plantarflexion angle at push-off & $-3.6 \pm 3.9$ & $-2.7 \pm 4.1$ & 0.205 \\
\hline Ankle plantarflexion angle at initial contact & & $0.9 \pm 0.3$ & 0.426 \\
\hline Kinetics & $0.9 \pm 0.4$ & $1.1 \pm 0.7$ & 0.234 \\
\hline Maximum ankle moment (Nm/kg) & $1.2 \pm 6.9$ & \\
\hline Maximum ankle power generation (W/kg) &
\end{tabular}

Table 4. Comparison of postoperative kinetic and kinematic parameters between groups. Negative values indicate hip extension and ankle plantarflexion. ${ }^{\star} P<0.05$.

Changes in temporospatial parameters. In the asymptomatic gait group, step width $(17.2 \pm 3.4 \mathrm{~cm}$ vs. $15.9 \pm 3.4 \mathrm{~cm}, P=0.003)$ and double-limb support duration $(13.4 \pm 3.7 \%$ vs. $11.7 \pm 4.3 \%, P=0.027)$ decreased after surgery, whereas stride length increased significantly $(105.2 \pm 11.5 \mathrm{~cm}$ vs. $109.1 \pm 15.2 \mathrm{~cm}, P=0.015)$. There were no changes in velocity $(P=0.317)$ and duration of the standing phase $(P=0.552)$.

However, in the gait disturbance group, temporospatial parameters did not improve postoperatively $(P=0.225$, $0.119,0.107,0.257$, and 0.355 for step width, stride length, velocity, duration of the standing phase, and duration of double-limb support duration, respectively).

Comparison of kinetic and kinematic parameters between groups. Preoperatively, the gait disturbance group had a declined maximum knee flexion angle during the swing phase $\left(60.5 \pm 6.4^{\circ}\right.$ vs. $54.8 \pm 9.5^{\circ}$, $P=0.001)$ and ankle plantarflexion angle at push-off $\left(-12.2 \pm 7.4^{\circ}\right.$ vs. $\left.-6.5 \pm 6.5^{\circ}, P=0.001\right)$ compared to the asymptomatic gait group (Table 3 ).

After surgery, the gait disturbance group still demonstrated a less maximum knee flexion angle during the swing phase $\left(62.3 \pm 5.4^{\circ}\right.$ vs. $\left.58.2 \pm 9.7^{\circ}, P=0.004\right)$ and ankle plantarflexion angle at push-off $\left(-12.8 \pm 8.2^{\circ}\right.$ vs. $-8.3 \pm 8.1^{\circ}, P=0.002$ ) compared to the asymptomatic gait group (Table 4 ).

Changes in kinetic and kinematic parameters. In the asymptomatic gait group, there were no significant changes in the mean pelvic anterior tilt $(P=0.066)$, maximum hip extension angle $(P=0.625)$, maximum knee flexion angle $(P=0.062)$, maximum ankle moment $(P=0.798)$, and maximum ankle power generation $(P=0.287)$. However, the ankle plantarflexion angle at push-off increased significantly $\left(-12.2 \pm 7.4^{\circ}\right.$ vs. $\left.-12.8 \pm 8.2^{\circ}, P=0.042\right)$.

In the gait disturbance group, the mean pelvic anterior tilt $\left(9.9 \pm 8.2^{\circ}\right.$ vs. $\left.11.6 \pm 7.7^{\circ}, P=0.028\right)$ increased and the maximum hip extension angle decreased $\left(-2.9 \pm 13.9^{\circ}\right.$ vs. $\left.0.7 \pm 16.2^{\circ}, P=0.006\right)$ after surgery. The maximum knee flexion angle $\left(54.8 \pm 9.5^{\circ}\right.$ vs. $\left.58.2 \pm 9.7^{\circ}, P=0.005\right)$ and the ankle plantarflexion angle at push-off $\left(-6.5 \pm 6.5^{\circ}\right.$ vs. $\left.-8.3 \pm 8.1^{\circ}, P=0.039\right)$ increased significantly. After surgery, the maximum ankle moment increased significantly $(0.9 \pm 0.4 \mathrm{Nm} / \mathrm{kg}$ vs. $0.9 \pm 0.3 \mathrm{Nm} / \mathrm{kg}, P=0.024)$, whereas the maximum ankle power generation $(P=0.583)$ remained unchanged.

In both groups, the ankle plantarflexion at initial contact was noted preoperatively $\left(-3.0 \pm 4.3^{\circ}\right.$ in asymptomatic gait group vs. $-2.6 \pm 3.7^{\circ}$ in gait disturbance group, $\left.P=0.502\right)$ and postoperatively $\left(-3.6 \pm 3.9^{\circ}\right.$ in asymptomatic gait group vs. $-2.7 \pm 4.1^{\circ}$ in gait disturbance group, $\left.P=0.205\right)$ (Figure 1 ). This ankle plantarflexion at initial contact did not change in both the asymptomatic gait $(P=0.377)$ and the gait disturbance $(P=0.851)$ groups by operation. 

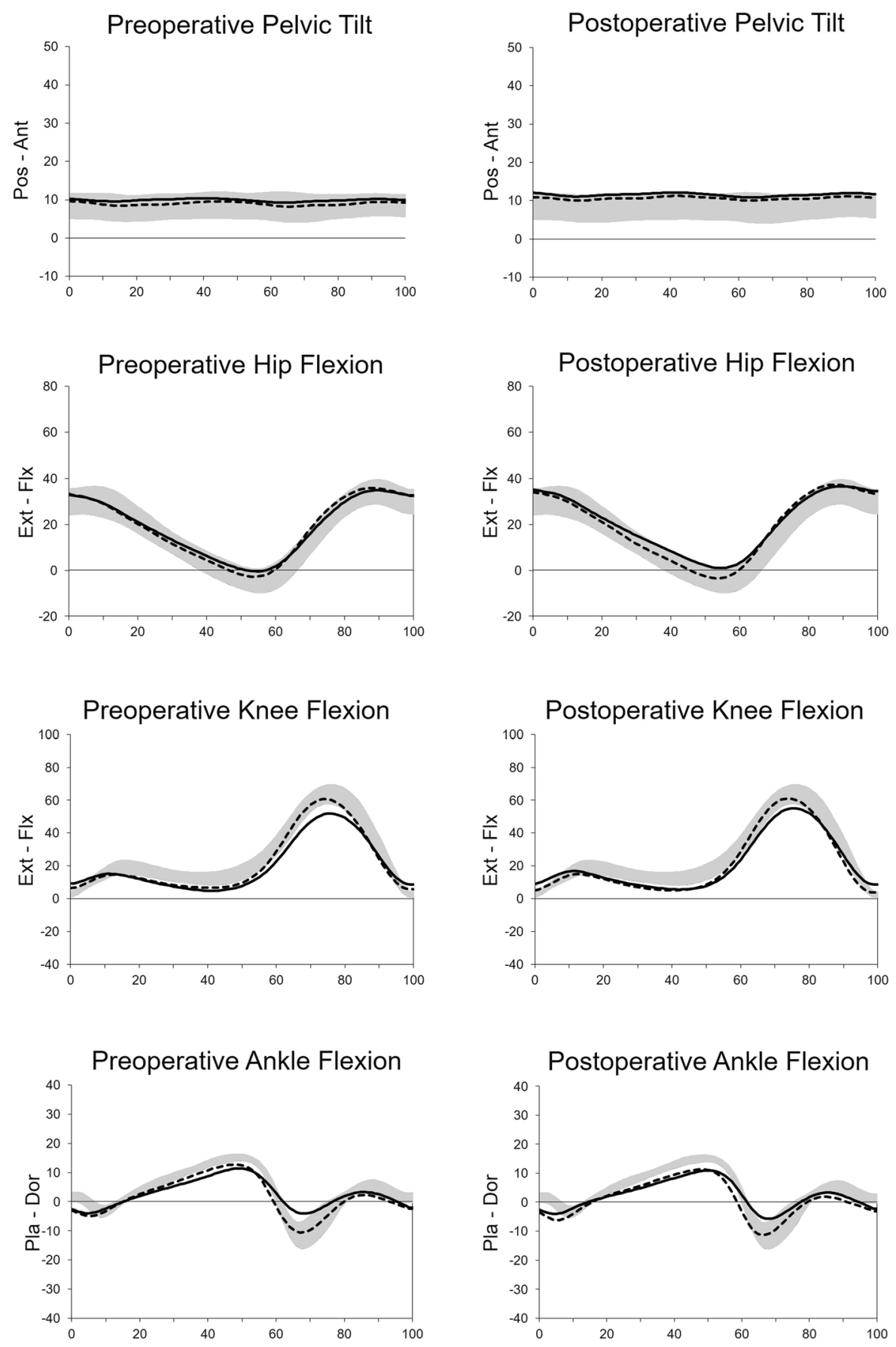

\section{Asymptomatic gait ------- Gait disturbance}

Figure 1. Kinematic graphs of the pelvis, hip, knee, and ankle before and after surgery. Preoperatively, knee flexion during the swing phase and ankle plantarflexion at push-off were decreased in the gait disturbance group compared to the asymptomatic gait group. These factors improved after surgery, but remained lower than in the asymptomatic gait group. In both groups, the ankle angle at initial contact demonstrated plantarflexion both before and after surgery. Ant anterior, Dor dorsiflexion, Ext extension, Flx flexion, Pla plantarflexion, Pos posterior.

\section{Discussion}

The natural progression of DCM is variable, but typically manifests as a slow, stepwise worsening of symptoms ${ }^{15}$. Previous studies have addressed the importance of early surgery to obtain substantial postoperative 
improvement ${ }^{2,13}$. Early diagnosis is difficult, as DCM is often painless at onset; as a result, DCM may not be diagnosed until it progresses to a late stage. Myelopathic gait is one of the DCM symptoms ${ }^{16}$, but many patients do not also exhibit a definite gait abnormality. Radcliff et al. ${ }^{14}$ found that $18 \%$ of patients with hip fracture manifested clinical findings consistent with DCM. Other studies reported an association between myelopathic gait and an increased risk of falling or subsequent hip (and other) fragility fractures ${ }^{17,18}$. Early detection of gait disturbances in DCM is important, not only for better prognosis of DCM after surgery, but also to prevent fractures related to the gait disturbance and to maintain the quality of life. In this study, we evaluated the subclinical myelopathic gait preoperatively and the improvements of gait abnormalities after surgery.

Previous studies reported reduced ankle plantarflexion and knee ROM as pathologic changes in kinematics, with decrease in gait speed and stride length and increase in double-limb support time and step width ${ }^{4,9,19-22}$. The gait disturbance group in this study also showed less knee and ankle ROM with a declined temporospatial parameters compared to the asymptomatic gait group. However, the decrease in knee flexion was not definite in the asymptomatic gait group, and the maximum knee flexion did not increase postoperatively. Instead, step width and double-limb support duration decreased, and stride length increased with the increase of ankle plantarflexion at push-off after surgery only in the asymptomatic gait group. Nagai et al. ${ }^{23}$ proposed gait speed and stride length as indices for evaluating progressive gait abnormalities. Considering the gait parameter improvement in the asymptomatic gait group, an increase of step width and double-limb support duration and a reduced stride length may be early signs of DCM. However, reduced gait speed or stride length may also reflect a patient's comfortable gait speed, which may be influenced by aging or the patient's preference. Comparing the gait patterns of people with untreated DCM to those of age- and gender-matched healthy controls, Malone et al. ${ }^{5}$ found that key differences exist in the motor strategies used in the terminal stance phase of gait (including peak ankle plantarflexion), which cannot be explained by speed alone. An increase of step width and double-limb support duration and a decrease in ankle push-off at terminal stance indicate early gait deterioration in patients with DCM.

Another abnormal kinematic finding in the asymptomatic gait group was ankle plantarflexion at initial contact. The ankle plantarflexion at initial contact was noted in both groups pre- and postoperatively. This abnormal ankle plantarflexion may be misdiagnosed as a foot drop that could be seen in patients with lumbar spinal disease. However, patients with foot drop due to weakness in ankle dorsiflexor walk with increased knee flexion for foot clearance. In patients with DCM, preoperative knee flexion was decreased, postoperative gait analysis showed improved knee flexion and an increase in stable foot clearance with a more powerful push-off demonstrating smooth and coordinated movement. Myelopathic gait is classically described as a "spastic pattern gait" and is accompanied by hyperreflexia and incoordination of agonist/antagonist muscles ${ }^{12,24}$. However, Malone et al. ${ }^{24}$ concluded the hyper-excitability of the stretch reflex did not contribute to the abnormal kinetic and kinematics. They suggested that paresis and poor proprioception were associated with gait impairment. Paresis may be related to the decrease of ankle plantarflexion at push-off. Proprioception plays an important role in smooth, coordinated activation of the extremities. Sensory information for proprioception is collected at the nerve endings and neural impulses subsequently travel toward the cerebral cortex via the posterior columns of the spinal $\operatorname{cord}^{22,25}$. Any disorder within these neural pathways, such as DCM, can impair proprioception. This may manifest as reduced knee flexion during swing phase known as spastic gait. In our opinion, ankle plantarflexion at initial contact may be another early sign of spastic gait related to the impaired proprioception.

Improvement in walking speed, step length, and knee ROM have been reported after DCM surgery ${ }^{11,26}$. Although we observed significant improvements in temporospatial parameters in the asymptomatic gait group, the gait disturbance group exhibited no significant improvements in these temporospatial parameters, despite improvements in kinetic and kinematic parameters. Furthermore, step width improved only in the asymptomatic gait group, which was relatively worse in the gait disturbance group postoperatively, although there was no difference preoperatively. The importance of early decompressive surgery has been previously discussed ${ }^{8,12,13}$. Malone et al. ${ }^{12}$ found no significant changes in temporospatial parameters at 1 year after surgery for DCM. GarzaRamos et al. ${ }^{8}$ concluded that patients with a higher preoperative Nurick grade with symptoms for more than 12 months may have significantly lower odds of gait improvement after surgery. In our gait disturbance group, the average duration of symptoms before surgery was 12.3 months. Furthermore, postoperative parameters in the gait disturbance group remained worse than those in the asymptomatic gait group. Early surgery appears mandatory for recovery of gait impairment.

Several qualitative functional outcome scales (e.g., JOA score, Nurick grade, European Myelopathy Score, and Myelopathy Disability Index scale) have been used clinically to assess the results of DCM surgery. However, in addition to having low sensitivity, these outcome scales are subjective, categorical, and overly simplified. In this study, lower limb motor function grade according to JOA did not demonstrate a significant change, even though the temporospatial parameters in the asymptomatic gait group and kinematic parameters in the gait disturbance group were improved. These results suggest that the quantitative assessment of spasticity and deterioration of proprioception, based on gait analysis, could play an important role in early detection of gait disturbances in patients with DCM, as well as in the assessment of postoperative improvements. Decompressive surgery after early detection of DCM is expected to reduce complications associated with gait disturbances, such as fragility fractures, and improve the patients' quality of life.

This study had several limitations. First, it had a retrospective design, and the operative methods differed between anterior and posterior decompression procedures. However, the surgical approach and the duration of symptoms were not significantly different between the asymptomatic gait and gait disturbance groups. Second, we only selected patients who could walk at least $20 \mathrm{~m}$ on their own; therefore, patients with severe gait disturbance or those who could only walk with an assistive device were not evaluated. Even when the patients are non-ambulatory, improved walking ability may be observed after surgery ${ }^{13}$. In this study, there was no significant improvement in the temporospatial parameters of the gait disturbance group; therefore, other study protocol besides the gait analysis should be added for the severely affected patients in the future. Finally, we set 
the end-point of postoperative improvement at 6 months considering the presence of other degenerative diseases, such as osteoarthritis or spinal stenosis, in this elderly population. This follow-up period was also based on previous studies ${ }^{26,27}$. A prospective study involving a larger number of patients (including non-ambulatory patients) and a longer follow-up period would be helpful to further understand the potential improvements in myelopathic gait after DCM surgery.

In conclusion, reduced ankle plantarflexion angle at push-off, increased step width, and increased doublelimb support duration may be early signs of DCM. Ankle plantarflexion at initial contact may also indicate early gait deterioration in patients with DCM. Even the patients without gait disability can be benefited from surgery in terms of the temporospatial parameter improvement. In the gait disturbance group, knee ROM and ankle plantarflexion at push-off improved after surgery, but were still lower compared to the asymptomatic gait group. Furthermore, preoperatively declined temporospatial parameters did not improve. Early diagnosis and surgery for DCM may be essential for preserving gait function and improving gait disability.

\section{Methods}

Study design and materials. This retrospective study was approved by the Asan Medical Center Institutional Review Board, and was conducted in accordance with the Declaration of Helsinki. Informed consent waiver was obtained from the Asan Medical Center Institutional Review Board (2020-1030). We reviewed the patients who had undergone decompressive surgery for DCM by a single surgeon (D.H.L) between 2014 and 2016. The following inclusion criteria were applied in this study: (1) aged 18 years and older, (2) clinical and radiological evidence of DCM, (3) able to walk at least $20 \mathrm{~m}$ without assistance from another person or a walking aid, and (4) availability of complete preoperative and postoperative three-dimensional gait analysis. Patients were excluded if they had thoracic or lumbar spinal disease, as well as other conditions that may affect their walking ability (e.g., leg length discrepancy more than $2 \mathrm{~cm}$, degenerative or rheumatoid osteoarthritis, peripheral nerve disease or injury, stiff joint, cardiac disease, respiratory disease, stroke, traumatic brain injury, and myelitis), or had a history of orthopedic surgery or neurosurgery that could affect gait. Sixty-two patients (46 males and 16 females) with a mean age of 58.6 years (range 42 to 76 years) were included in this study.

Since gait analysis was impossible to determine for patients who could not walk independently, all of the included patients had their lower limb motor function graded as 2, 3, or 4, according to the Japanese Orthopedic Association (JOA). Patients with grade 4 ("normal") were classified as the asymptomatic gait group $(\mathrm{n}=30)$, and those with grade 3 ["possible to walk without cane or aid, but slow" $(\mathrm{n}=17)$ ] or grade 2 ["need cane or aid only on stairs" $(n=15)]$ were combined and classified as the gait disturbance group $(n=32)$.

Gait analysis protocol. Gait analysis was performed on a flat-ground, 20-m track using a computerized three-dimensional gait analysis system consisting of six infrared cameras (Motion Analysis ${ }^{\oplus}$, Santa Rosa, CA, USA). Fifteen retroreflective markers were attached to anatomical landmarks using the Modified Helen Hayes method. The capture volume of the cameras was calibrated prior to each assessment to achieve calibration residuals less than $2.5 \mathrm{~mm}$. Anthropometric data were collected according to a standard protocol. After a warm-up trial, individuals walked continuously and freely along the track five times. Participants were permitted to rest between trials to avoid fatigue. Two force-plates, located under the path, recorded ground reaction forces during walking, and joint moments were expressed as internal moments to counter the ground reaction force. Kinematic data was shown as the angle between two segment axes projected into the each sagittal, coronal, and transverse plane. The gait cycle events (heel strike and toe off) were identified automatically from the force plate data during walking. The kinematic and kinetic data were derived for pelvis, hip, knee, and ankle in three planes.

The following parameters from kinematic and kinetic data were analyzed: temporospatial parameters (step width, stride length, velocity, standing phase duration, and double-limb support duration), kinematic parameters (mean pelvic anterior tilt, maximum hip extension angle, maximum knee flexion angle, ankle plantarflexion angle at push-off, and ankle plantarflexion angle at initial contact), and kinetic parameters (maximum ankle moment and maximum ankle power generation).

Gait analysis was performed before surgery and at 6 months after surgery. This postoperative period was a compromise between increasing the likelihood of optimal surgical recovery and reducing the potential confounding effects of aging and degenerative joint disease during follow-up ${ }^{27}$.

Statistical analysis. Statistical analysis was performed using the SPSS version 23 (IBM Corp, Armonk, $\mathrm{NY}$ ). Independent $t$-tests were used to compare the parameters between asymptomatic gait and gait disturbance groups, and paired t-tests were used to compare the parameters between preoperative and postoperative evaluations. Values are presented as mean and ranges. The statistical significance level was set at $P<0.05$.

Ethical approval. This study was approved by the Asan Medical Center Institutional Review Board (IRB No. 2020-1030).

\section{Data availability}

The datasets generated during and/or analyzed in the current study are available from the corresponding author upon reasonable request.

Received: 25 February 2021; Accepted: 18 May 2021

Published online: 27 May 2021 


\section{References}

1. Nouri, A., Tetreault, L., Singh, A., Karadimas, S. K. \& Fehlings, M. G. Degenerative cervical myelopathy: Epidemiology, genetics, and pathogenesis. Spine (Phila Pa 1976) 40, E675-E693 (2015).

2. Cheung, J. P. Y., Cheung, P. W. H., Chiu, C. K., Chan, C. Y. W. \& Kwan, M. K. Variations in practice among Asia-Pacific surgeons and recommendations for managing cervical myelopathy: The first Asia-Pacific spine society collaborative study. Asian Spine J. 13, 45-55 (2019).

3. Yamada, T. et al. Surgical outcomes for lumbar spinal canal stenosis with coexisting cervical stenosis (tandem spinal stenosis): A retrospective analysis of 565 cases. J. Orthop. Surg. Res. 13, 60 (2018).

4. Yoon, J. Y., Chang, H., Park, K. B., Lee, S. J. \& Choi, B. W. Effect of the number of involved spinal cord segments on gait function in patients with cervical spondylotic myelopathy. Asian Spine J. 6, 233-240 (2012).

5. Malone, A., Meldrum, D. \& Bolger, C. Gait impairment in cervical spondylotic myelopathy: Comparison with age- and gendermatched healthy controls. Eur. Spine J. 21, 2456-2466 (2012).

6. Singh, A. \& Crockard, H. A. Quantitative assessment of cervical spondylotic myelopathy by a simple walking test. Lancet 354, 370-373 (1999).

7. Kalsi-Ryan, S. et al. Characteristics of upper limb impairment related to degenerative cervical myelopathy: Development of a sensitive hand assessment (graded redefined assessment of strength, sensibility, and prehension version myelopathy). Neurosurgery 3 , E292-E299 (2020).

8. De la Garza-Ramos, R. et al. Prognostic value of preoperative Nurick grade and time with symptoms in patients with cervical myelopathy and gait impairment. World Neurosurg. 105, 314-320 (2017).

9. Siasios, I. D. et al. The role of gait analysis in the evaluation of patients with cervical myelopathy: A literature review study. World Neurosurg. 101, 275-282 (2017).

10. Maezawa, Y., Uchida, K. \& Baba, H. Gait analysis of spastic walking in patients with cervical compressive myelopathy. J. Orthop. Sci. 6, 378-384 (2001).

11. Moorthy, R. K., Bhattacharji, S., Thayumanasamy, G. \& Rajshekhar, V. Quantitative changes in gait parameters after central corpectomy for cervical spondylotic myelopathy. J. Neurosurg. Spine 2, 418-424 (2005).

12. Malone, A., Meldrum, D. \& Bolger, C. Three-dimensional gait analysis outcomes at 1 year following decompressive surgery for cervical spondylotic myelopathy. Eur. Spine J. 24, 48-56 (2014).

13. Takeoka, Y. et al. Postoperative walking ability of non-Ambulatory cervical myelopathy patients. Spine (Phila Pa 1976) 43, E927E934 (2018).

14. Radcliff, K. E. et al. High incidence of undiagnosed cervical myelopathy in patients with hip fracture compared with controls. J. Orthop. Trauma 30, 189-193 (2016).

15. Matz, P. G. et al. The natural history of cervical spondylotic myelopathy. J. Neurosurg. Spine 11, 104-111 (2009).

16. Baker, J. M. Gait Disorders. Am. J. Med. 131, 602-607 (2018).

17. Horowitz, J. A. et al. Fragility fracture risk in elderly patients with cervical myelopathy. Spine (Phila Pa 1976) 44, 96-102 (2019).

18. Kimura, A. et al. Fall-related deterioration of subjective symptoms in patients with cervical myelopathy. Spine (Phila Pa 1976) 42, E398-E403 (2017).

19. Haddas, R. et al. Spine and lower extremity kinematics during gait in patients with cervical spondylotic myelopathy. Spine J. 18, $1645-1652$ (2018).

20. Kim, C. R., Yoo, J. Y., Lee, S. H., Lee, D. H. \& Rhim, S. C. Gait analysis for evaluating the relationship between increased signal intensity on $\mathrm{t} 2$-weighted magnetic resonance imaging and gait function in cervical spondylotic myelopathy. Arch. Phys. Med. Rehabil. 91, 1587-1592 (2010).

21. Lee, J. H., Lee, S. H. \& Seo, I. S. The characteristics of gait disturbance and its relationship with posterior tibial somatosensory evoked potentials in patients with cervical myelopathy. Spine (Phila Pa 1976) 36, E524-E530 (2011).

22. Takayama, H. et al. Impaired joint proprioception in patients with cervical myelopathy. Spine (Phila Pa 1976) 30, 83-86 (2005).

23. Nagai, T. et al. Analysis of spastic gait in cervical myelopathy: Linking compression ratio to spatiotemporal and pedobarographic parameters. Gait Posture 59, 152-156 (2018).

24. Malone, A., Meldrum, D., Gleeson, J. \& Bolger, C. Electromyographic characteristics of gait impairment in cervical spondylotic myelopathy. Eur. Spine J. 22, 2538-2544 (2013).

25. Grob, K. R., Kuster, M. S., Higgins, S. A., Lloyd, D. G. \& Yata, H. Lack of correlation between different measurements of proprioception in the knee. J. Bone Joint Surg. Br. 84, 614-618 (2002).

26. Haddas, R. et al. Effect of cervical decompression surgery on gait in adult cervical spondylotic myelopathy patients. Clin. Spine Surg. 31, 435-440 (2018).

27. Pandita, N. et al. Neurological recovery pattern in cervical spondylotic myelopathy after anterior surgery: A prospective study with literature review. Asian Spine J. 13, 423-431 (2019).

\section{Author contributions}

D.H.L. and J.K.H. designed the research. K.B.P. and J.Y.Y. performed the analysis. D.H.L. and K.B.P. drafted and revised the manuscript. J.H.C., C.J.H., and J.K.H. collected the data. J.H.C., C.J.H., C.S.L., and C.K. supervised and reviewed the manuscript. All authors have approved the final version of the manuscript.

\section{Funding}

No fund, grant, or other support was received.

\section{Competing interests}

The authors declare no competing interests.

\section{Additional information}

Correspondence and requests for materials should be addressed to K.-B.P.

Reprints and permissions information is available at www.nature.com/reprints.

Publisher's note Springer Nature remains neutral with regard to jurisdictional claims in published maps and institutional affiliations. 
(c) (i) Open Access This article is licensed under a Creative Commons Attribution 4.0 International cc) License, which permits use, sharing, adaptation, distribution and reproduction in any medium or format, as long as you give appropriate credit to the original author(s) and the source, provide a link to the Creative Commons licence, and indicate if changes were made. The images or other third party material in this article are included in the article's Creative Commons licence, unless indicated otherwise in a credit line to the material. If material is not included in the article's Creative Commons licence and your intended use is not permitted by statutory regulation or exceeds the permitted use, you will need to obtain permission directly from the copyright holder. To view a copy of this licence, visit http://creativecommons.org/licenses/by/4.0/.

(C) The Author(s) 2021 